\title{
LA MESA REDONDA DE ECONOMISTAS FINANCIEROS
}

\section{THE FINANCIAL ECONOMISTS ROUNDTABLE}

Carlos Díaz Gómez: Universidad de Alcalá de Henares. Madrid (España) carlos_diazgomez@yahoo.com

\section{CURRÍCULUM VITAE}

Doctor en Filosofía por la Universidad Complutense de Madrid (España) con Premio Extraordinario, Licenciado en Derecho por la Universidad Complutense de Madrid (España) con Premio Extraordinario, Licenciado en Altos Estudios Constitucionales con Premio Extraordinario y Profesor de la Universidad Complutense de Madrid (España). Autor de numerosos libros y artículos en revistas especializadas y universitarias.

\section{RESUMEN}

En la Mesa Redonda de Economistas Financieros de 2005 se discutió sobre los fondos de cobertura. Un fondo de cobertura es una asociación privada para inversión, normalmente abierta sólo a individuos adinerados e inversores institucionales cualificados. La asociación la dirige un socio general que supervisa las actividades de inversión del fondo. Tales fondos tienen una considerable libertad en sus estrategias de inversión. En los últimos años han crecido, pero siguen generando muchos problemas. 


\section{PALABRAS CLAVE}

Mesa Redonda - Economistas - Fondos de cobertura - Problemas

\section{ABSTRACT}

The Financial Economists Roundtable, in 2005, was discussed on hedge funds. A hedge fund is a private investment, normally open only to wealthy individuals and qualified institutional investors. The association is headed by a general partner who oversees the fund's investment activities. Such funds have considerable freedom in their investment strategies. In recent years they have grown, but still generate many problems.

\section{KEY WORDS}

Roundtable - Economists - Hedge Funds - Problems

TEXTO:

La Mesa Redonda de Economistas Financieros (FER, según sus siglas en inglés) es un grupo de economistas financieros de alto rango que han contribuido de forma significativa a la literatura financiera y pretenden enriquecer con sus conocimientos el debate político actual. La Mesa Redonda se centra en aspectos macroeconómicos de las inversiones, finanzas corporativas e instituciones y mercados financieros, tanto en los Estados Unidos como internacionalmente. 
La MREF se creó en 1993 y se reúne todos los años. Los miembros que asisten a las sesiones de la Mesa discuten aspectos concretos de política, sobre los que pueden eventualmente pronunciarse haciendo público un comunicado. Cuando se emite un comunicado, refleja un consenso entre la mayoría de los miembros asistentes y es firmado por quienes lo suscriben. Los comunicados pretenden que los responsables de la política pública tengan mayor conciencia y entiendan mejor todas estas cuestiones.

\section{Comunicado de la Mesa Redonda de Economistas Financieros sobre Fondos de cobertura}

(3 de noviembre de 2005)

Stanford, California. La Mesa Redonda de Economistas Financieros (MREF) discutió los fondos de cobertura en su reunión anual de 2005 y preparó el siguiente comunicado.

\section{$\underline{\text { Resumen ejecutivo }}$}

Los fondos de cobertura han crecido rápidamente en los últimos años y hoy suponen aproximadamente un octavo del total de los fondos mutuos. Mas estas sociedades comanditarias suscitan una serie de problemas. Sus gastos de administración son muy elevados y sus estrategias de inversión suelen ser muy arriesgadas, con una pequeña probabilidad de pérdidas muy importantes. No todos los inversores comprenden estos y otros riesgos. En respuesta a estas circunstancias, la Mesa Redonda de Economistas Financieros recomienda que se limite la cantidad que pueden invertir los fiduciarios de pequeños inversores a un modesto porcentaje de los activos que administran, que los reguladores se abstengan de rescatar a fondos con problemas, y que se estandaricen las medidas de los resultados y de los riesgos 
de los distintos fondos. Sin perjuicio de la cláusula caveat emptor ("cuídese el comprador"), es importante conseguir un comportamiento mejor fundado y más racional por parte del inversor.

\section{Introducción}

Un fondo de cobertura es típicamente una sociedad comanditaria, sociedad mercantil de carácter personalista, en gran medida no sujeta a regulación, cuyos socios comanditarios son particulares ricos e inversores institucionales y cuyo administrador/asesor de inversiones es un socio colectivo. Un fondo de cobertura puede apalancarse, amplificando de este modo la variabilidad de sus resultados. La redención de las cantidades invertidas se halla sujeta a restricciones, de modo que se trata de inversiones en gran medida ilíquidas. El primer fondo de cobertura del que se tiene noticia se instauró en 1949 (NOTA 1), y adoptó una estrategia accionaria larga/corta. No fue hasta mediados de los años ochenta cuando los fondos de cobertura adquirieron importancia. Antes de 1985, existían aproximadamente 40 fondos de cobertura. Su número aumentó hasta más de mil en 1995 y hasta unos 8.000 en 2005. Actualmente, los fondos de cobertura administran un millón de millones de dólares, aproximadamente un octavo de la cantidad administrada por los fondos mutuos.

\section{A. Observaciones e inquietudes}

1. Comprender los rendimientos, los gastos y el riesgo. Existe preocupación por el hecho de que los inversores en fondos de cobertura puedan no comprender del todo su auténtica rentabilidad ni los riesgos que les afectan. Los gastos son elevados. La comisión de administración que percibe el socio colectivo normalmente es del 1 ó del 2 por ciento de los activos administrados, pagadera anualmente, y suele existir también una tarifa asimétrica adicional ligada al rendimiento logrado. Esta comisión 
de incentivo, o de participación en cuenta, suele ser del 20 por ciento, y con frecuencia se estructura de modo que sólo haya de pagarse si la rentabilidad acumulada excede de un umbral de rentabilidad, conocido como "nivel máximo del agua". Cuando la rentabilidad acumulada no alcanza esta marca, el socio colectivo puede cerrar el fondo, y crear uno nuevo con el fin de fijar una nueva marca básica a partir de la cual generar comisiones ligadas a sus resultados. La estructura asimétrica de las comisiones presenta al socio colectivo un incentivo para que acometa estrategias de inversión de alto riesgo, ya que puede lograr una rentabilidad importante si acierta en su estrategia, pero no se expone a sufrir las pérdidas si su estrategia fracasa. El hecho de que los inversores insistan en que los socios colectivos inviertan ellos mismos en el fondo compensa en cierto grado tal incentivo. No obstante, la vida media de un fondo de cobertura no suele exceder de los tres años.

Las rentabilidades de las estrategias de muchos fondos de cobertura no se distribuyen normalmente, sino que su distribución se caracteriza por tener colas gruesas. Hay quien se refiere a este riesgo como el "problema del peso". Esto es, durante un periodo muy largo el gobierno mexicano no devalúa el peso, para devaluarlo luego de golpe, de modo que los tenedores de pesos sufren pérdidas muy importantes. Dicho de otro modo, día a día, la probabilidad de una gran pérdida es reducida. El riesgo de cola hace que las medidas estándares de la volatilidad, la rentabilidad y el rendimiento del fondo, tales como la ratio de Sharpe (NOTA 2), no sean una guía adecuada para orientar a los inversores. Por su propia naturaleza, el riesgo de cola no es fácil de medir. Además, la rentabilidad media tras computar el riesgo tiende a estar exagerada, por el sesgo de supervivencia y por otros problemas derivados de los informes y de los datos, dificultando la comparación del rendimiento de los fondos de cobertura con otras alternativas de inversión. Otro de los riesgos es la iliquidez asociada con las posiciones concretas que toman los fondos de cobertura. 
Los inversores, particularmente el pequeño inversor y sus agentes, han de ser cautos; los datos disponibles sobre los rendimientos hacen que sea difícil juzgar la auténtica rentabilidad de los fondos de cobertura y el riesgo de estos vehículos de elevado coste. Si bien la reputación puede servir de mecanismo disciplinario, no siempre ha sido eficaz. Para que se entienda, es preciso que el inversor tenga muchísimo cuidado.

2. Riesgo sistémico. Por riesgo sistémico entendemos el riesgo de que el incumplimiento de una de las partes que intervienen en una transacción pueda provocar el incumplimiento de otras partes: A no puede pagar a B, quien no puede entonces pagar a C, y así sucesivamente. La MREF cree que un riesgo sistémico con carácter de efecto dominó capaz de poner en peligro a las instituciones financieras es hoy pequeño, pero reconocemos la inherente dificultad de extraer en este sentido conclusión concluyente alguna. Más recientemente, retrasos en el procesamiento de las órdenes por parte de las propias instituciones financieras han hecho que sea difícil para los fondos de cobertura saber exactamente cuáles son sus posiciones en tiempo real. Las partes que participan en una transacción no se conocen entre sí, y esta incertidumbre, unida al riesgo de cola, es motivo de preocupación para los inversores que pretenden comprender cuál es exactamente y en qué medida les puede afectar el posible riesgo de que todo el sistema se venga abajo como un dominó. La dificultad de valorar la exposición potencial al riesgo sistémico hace más importante la necesidad de extremar la prudencia a la hora de decidir qué parte de una cartera se dedica a invertir en fondos de cobertura. En ciertas ocasiones, la liquidez puede verse congelada temporalmente en ciertos mercados a consecuencia de la actividad que generan los fondos de cobertura. Sin embargo, desde el episodio del Long Term Capital Management (LTCM) de 1998, muchos fondos de cobertura se han vuelto más cautos en su elección de la otra parte con la que cierran un trato, y no hay hoy ningún fondo concreto de tamaño relativo al mercado similar al que en su día tenía LTCM. Además, actualmente los reguladores bancarios siguen mucho más 
de cerca la exposición crediticia y la exposición respecto de todas las partes involucradas en las operaciones que las instituciones financieras realizan con fondos de cobertura.

3. Fondo de fondos. Los fondos de fondos (de cobertura) pueden cumplir un útil papel aportando información y disciplina al mercado. Un fondo de fondos asigna capital entre una serie de fondos de cobertura individuales, poniendo al alcance de los inversores a gestores de los que no podrían disponer en otro caso, así como diversificación en cuanto al estilo y a la ley de los grandes números. Por tales servicios, un fondo de fondos carga (naturalmente) comisiones adicionales de gestión y de incentivos de hasta otro 50 por ciento de las comisiones correspondientes a los fondos subyacentes. Este coste añadido ha de evaluarse en relación a la eficiencia de la información y la disciplina que puedan aportar al proceso. Algunos de nosotros sospechamos que los servicios provistos por algunos de estos fondos de fondos valen su coste, y que aumentan la eficiencia del mercado. Otros de nosotros creemos que con 8.000 fondos de cobertura compitiendo unos con otros, en muchas ocasiones con idénticas estrategias de inversión, ha de haber necesariamente perdedores, en particular cuando se consideran los elevados costes. Todos nosotros creemos que los fondos de fondos de fondos, los F3, que invierten en fondos de fondos, no tienen una ratio de coste / beneficio favorable.

\section{B. Recomendaciones}

1. Los administradores fiduciarios deberían limitar cuidadosamente sus inversiones en fondos de cobertura. Habida cuenta de los riesgos de cola y de salida que, junto con la falta de transparencia, son propios de este tipo de inversiones, la MREF ve con preocupación que pueda no ser apropiada una gran exposición por parte de los fondos de pensiones y otros inversores fiduciarios que invierten en nombre de terceros, concretamente pequeños inversores. El reciente fraude del fondo de 
cobertura Bayou pone de manifiesto lo que puede ir mal. Los gestores de fondos se enfrentan a conflictos de incentivos que pueden impedirles defender adecuadamente los intereses de los beneficiarios cuyos fondos les han sido confiados. La dificultad que conlleva valorar toda la diversidad de riesgos que afectan a los fondos de cobertura debería bastar para que se limitase la cantidad invertida en fondos de cobertura a una modesta fracción de los activos totales administrados. La MREF se teme que los consejos de administración de algunos administradores fiduciarios, concretamente aquellos compuestos mayoritariamente por no profesionales, puedan no comprender cabalmente la auténtica rentabilidad, riesgos y costes asociados con la inversión en fondos de cobertura.

2. Los reguladores deberían comprometerse muy seriamente a no rescatar fondos de cobertura. La MREF cree que los reguladores bancarios no deberían rescatar a los fondos de cobertura. Ni uno ni dos de estos fondos suponen un riesgo sistémico, si bien una quiebra individual podría perturbar de modo temporal seriamente el mercado. La existencia cierta de un seguro de rescate gratuito por parte del gobierno crea incentivos negativos que invitan a un comportamiento especulativo. Hacer explícita una política contraria al rescate reduciría tales incentivos. Si bien el riesgo de cola es un problema, no prevemos escenario alguno en que la autoridad monetaria se vea en la obligación de intervenir en su capacidad de prestamista de última instancia.

3. Debería estandarizarse el rendimiento y las medidas del riesgo. La MREF recomienda que instituciones tales como el Instituto de auditores financieros (NOTA 3) o la Asociación alternativa de analistas de inversión colegiados (NOTA 4) elaboren estándares para medir el rendimiento y el riesgo de los fondos de cobertura, como se ha hecho para otros vehículos de inversión. Es decir, deberíamos contar con medidas estandarizadas referidas a rentabilidades brutas y netas, ratios de gastos, apalancamiento, volatilidad de la rentabilidad, riesgo de crédito y de liquidez. Si bien 
algunos fondos de cobertura gozan de la reputación de haber elaborado buenas medidas internas de riesgo, no las han puesto a disposición de los inversores. Mejores medidas del riesgo y la rentabilidad posibilitarían una mejor comprensión y una inversión racional. Permitirían comparaciones más uniformes entre fondos de cobertura. Por último, la MREF anima que se investigue la estructura de comisiones asimétricas y sus efectos sobre el comportamiento inversor de los fondos de cobertura.

La adopción de estas recomendaciones habría de redundar en condiciones más favorables para los fondos de cobertura, y traducirse en una mejor comprensión del rendimiento, los gastos y los riesgos. Albergamos la esperanza de que la industria facilite voluntariamente información más estandarizada. Aunque no quepa prescindir de la cláusula caveat emptor, habría de venir seguida de un comportamiento inversor más racional.

\section{LA MESA REDONDA DE ECONOMISTAS FINANCIEROS \\ (Miembros firmantes del comunicado)}

-Rashad Abdel-Khali

Universidad de Illinois

www.uillinois.edu

-George Kaufman

Loyola University Chicago

www.luc.edu

-Edgard Altman

New York University

www.nyu.edu

-Dennis Logue

University of Oklahoma 
www.ou.edu

-Harold Bierman

Cornell University

www.cornell.edu

-Jay Ritter

University of Florida

www.ufl.edu

-Marshall Blume

University of Pennsylvania

www.upenn.edu

-Stephen Schaefer

London Business School

www.london.edu

-Willard Carleton

University of Arizona

www.arizona.edu

-Eduardo S. Schwartz

University of California at Los Angeles

www.anderson.ucla.edu

-Kenneth Scott

Stanford University

www.stanford.edu

-Andrew Chen

Southern Methodist University

www.smu.edu

-Tom Copeland

Monitor Group

WWW.monitor.com

-Lemma W. Senbet 
University of Maryland

www.umd.edu

-Elroy Dimson

London Business School

www.london.edu

-William F. Sharpe

Stanford University

www.stanford.edu

-Franklin Edwards

Columbia University

www.columbia.edu

-Jeremy Siegel

University of Pennsylvania

www.upenn.edu

-Seymour Smidt

Cornell University

www.cornell.edu

-Robert Eisenbeis

Federal Reserve Bank of Atlanta

www.frbatlanta.org

-Wayne Ferson

Boston College

www.bc.edu

-Robert Stambaugh

University of Pennsylvania

www.upenn.edu

-Laura Starks

University of Texas

www.utexas.edu 
-Charles Goodhart

London School of Economics

www.lse.ac.uk

-Richard Herring

University of Pennsylvania

www.upenn.edu

-Hans Stoll

Vanderbilt University

www.vanderbilt.edu

-Thomas Ho

Thomas Ho Company

www.thomasho.com

-Alan Kraus

University of British Columbia

www.ubc.ca

-James C. Van Horne

Stanford University

www.stanford.edu

-Randolph Westerfield

University of Southern California

www.usc.edu

-Edward Kane

Boston College

www.bc.edu

-J. Fred Weston

University of California at Los Angeles

www.ucla.edu 


\section{NOTAS:}

1. Keynes y sus socios ya invertían en derivados y se apalancaban tratando de cubrirse, tal y como puede leerse al principio del tomo XII de sus Obras Completas.

2. Forma de medir la rentabilidad tras considerar el riesgo. Es la relación entre el exceso de rentabilidad de la cartera y el riesgo de la misma, que viene medido por la desviación estándar de la rentabilidad de la cartera. El exceso de rentabilidad se calcula sobre un nivel de riesgo "cero", normalmente representado por una inversión en letras del Tesoro de los Estados Unidos.

\section{The CFA Institute}

4. The Chartered Alternative Investment Analyst Association 